\title{
Cytochrome Oxidase I based haplotype diversity of Mahseer (Tor tor) from eight sites in Southern Rajasthan
}

\author{
Sudha Summarwar ${ }^{1}$, K.P. Yadav ${ }^{2}$, Harendra Kumar ${ }^{1}$, and Satya tailor ${ }^{1}$ \\ ${ }^{1}$ Sangam University \\ ${ }^{2}$ Sangam University, Bhilwara
}

June 18, 2020

\begin{abstract}
Mahseer (Tor tor) is an important game fish in southern Rajasthan, India, and has recently been listed as an endangered species. The present study aimed to provide robust taxonomic classification of the species by calculating the haplotype gene frequency of Mahseer indigenous to the water bodies of southern Rajasthan. Thirty-two Tor tor samples were collected from eight sites in southern Rajasthan, India. The Cytochrome oxidase I (COI) gene was sequenced from all 32 samples. Haplotype frequencies of the samples collected were measured and phylogenetic analysis was performed. We observed that the Tor tor samples collected from the eight sites could be divided into three haplotypes, hap_1, hap_2, and hap_3. Samples collected from five sites (Chambal river, Rana Pratap Sagar, Bassi dam, Kali Sindh river, and Parwan river) exhibited the same COI haplotype, hap_1. Tor tor hap_2 was found only in samples from Badi lake; samples from Daya dam and Madar tank exhibited only Hap_3, giving a clear idea about phylogeny and group affiliations. This data can be used while repopulating the fish, which will contribute to better ecosystem management.
\end{abstract}

\section{Introduction}

Conservation of genetic diversity is critical for the continual survival of the fisheries industry and plays a pivotal role in the socio-economic development of our nation. Owing to the challenges associated with the ever-increasing demands for resources and the need to conserve fresh water bodies, the onus for ecological conservation now lies with local and regional biodiversity regulators and policy-makers. This demands a decisive shift from piecemeal steps to a comprehensive and practical multidisciplinary approach for conservation gambits (Adrian et al ., 2019). The fisheries segment provides employment to 14 million individuals in India, and a lack of species conservation could have serious ramifications on employment in the sector. Conservation plans to perk up the declining wild fish populations have been delayed by an unstable taxonomy.

Mahseer is a popular game and food fish of India. It provides unparalleled recreation to anglers from all over the world as a sport fish superior to the salmon. However, anthropogenic activities such as dam-building and over-fishing have adversely affected the Mahseerpopulation across the country. Once an abundant species, theMahseer population has been decreasing in natural waters and the different Mahseer species are now either categorized as "threatened" or "endangered". Moreover, $T$ tor has been recently classified as a data deficient species (Rayamajhi et al. 2018); there is, therefore, an urgent need to validate the conspecificity of the species from the river Mahananda (northern Bengal) with records of T. tor from other parts of India. However, the presence of only a few haplotypes of Mahseer species (Tor tor ), indiscernible morphological features, and paucity of research on accurate classification (Jayaram, 1999) have led to confusion in accurate taxonomic classification of this fish.

The advent of molecular biology and DNA markers has helped the progress of pisciculture by enabling the study of genetic variability, breeding patterns, institution of parentage identifying strain and species and 
high-resolution genetic linkage maps.

As genetic markers are important tools for the study of fish species and population characterization, the development of molecular methods has permitted genomic analysis and made the analysis of existing variations possible. Similarly, gene frequencies of mitochondrial genes are also indicative of phylogeny and close associations. Cytochrome oxidase I (COI), a mitochondrial gene, is a popular target for species identification and barcoding; it has been reported that approximately $98 \%$ of reported marine fish species can be identified by COIbarcoding. This approach has been employed to catalogue and record fish in many geographic regions (Asgharian et al. , 2011; Aquilinoet al. , 2011 and Cawthorn et al ., 2011). However, there is a dearth of genetic studies conducted on Mahseer species in India. Older studies have mainly explored taxonomy based only on morphological characteristics and therefore, there is a need to perform molecular studies [such as those by Nguyen et al. (2008)] to aid in the conservation of this endangered species. There is paucity of studies on Mahseer genetic structure reported across the Indian waters (Sati et al ., 2013) and in Rajasthan state. An examination of the mitochondrial genetic information of nine Mahseer species belonging to the Tor, Neolissochilus, and Naziritor genera was performed by Khare et al. in 2014. Their study highlighted the need for integrating molecular and morphological data to accomplish taxonomic revision of the Tor and Naziritor genera.

The mitochondrial genome has been effectively used for phylogenetic analysis and to solve taxonomic ambiguity in Mahseer species (Pavan-Kumar et al ., 2016). Therefore, we undertook this study to perform phylogenetic analysis on Mahseer species indigenous to southern Rajasthan using mitochondrial DNA sequences.

In the present study, we have analysed haplotype diversity ofMahseer (Tor sp.) from southern Rajasthan using the $C O I$ gene frequencies. Accurate taxonomic identification using molecular markers is an indispensable step towards the protection of this endangered fish.

\section{Materials and Methods}

Sample collection: A total of 32 samples of Tor tor were collected from eight different geographical sites in southern Rajasthan (Chambal River, Rana Pratap Sagar Dam, Bassi Dam, Kali Sindh River, Parwan River, Badi Lake, Daya Dam and Madar Tank; Figure 1). Fin tissues were collected by non-invasive techniques (Wasco et al.,2003) followed by antiseptic treatment of organism before they were reverted to their habitat. The fishes were handled according to the guidelines approved by the Agricultural Laboratory, Udaipur, Rajasthan, India. Caudal fin samples were preserved in 2 -ml vials containing $75 \%$ ethanol and stored at $-20^{\circ} \mathrm{C}$ until further processing.

DNA extraction: The DNA extraction, quantification, amplification, and authentication were carried out according to protocol proposed by Crandall et al. (1999). DNA extraction was performed using the phenolchloroform extraction protocol. DNA quantification was performed by two methods, using a Nano-Drop spectrophotometer (Implen) and agarose gel electrophoresis with HaeIII digested $\varphi 174$ RF DNA as a molecular marker (Abgene).

Capillary sequencing: The PCR amplicons were amplified using the forward and reverse primer in separate reactions. These amplicons were purified and sequenced in an automated DNA Sequencer (ABI PRISM 310 Genetic Analyzer Applied Biosystems). Sequence Analysis Software Version 5.2 (Applied Biosystems, CA, USA) was used to generate sequence trace files and contiguous read lengths. The standard protocol prescribed by ABI systems for operating ABI310 Genetic Analyzer was followed for sequencing the COI gene of the specimens.

Sequence Analysis: The raw DNA sequences were edited using BioEdit sequence alignment editor version 7.0.5.2. BLASTN program was used to compare the sequences retrieved from the two chromatograms, and fragments showing 100\% alignment with no gap or indels (insertion/deletions) were selected. The selected sequence fragments were aligned using ClustalX software. Finally, each sequence was compared using the NCBI BLASTN tool to examine the complete alignment with the partial coding sequence 
of fish mitochondrial COI gene. The sequences were translated using the online software ORF finder (http://www.ncbi.nlm.nih.gov/gorf/ gorf.html) and aligned using the NCBI BLASTP tool.

Haplotyping and phylogenetic analysis: A haplotype network was constructed using the medianjoining network method as implemented in NETWORK (version 4.1.1.1 available at www.fluxusengineering.com;Bandelt et al. 1999). This method begins by combining the minimum spanning trees (MSTs) within a single network (minimum spanning network, MSN) using an analogous algorithm to that proposed by Excoffier and Smouse (1994). Then, median vectors (which represent missing intermediates haplotypes) are added to the network using the parsimony criterion. Tajima's D test (Tajima 1989) and Fu's Fs test (Fu 1997) with 10,000 permutations as implemented in ARLEQUIN (version 3.01; Schneider et al., 2000) were used to test the hypothesis of neutrality of the marker. Negative Tajima's D-values can indicate selection, but also population bottlenecks or population expansions (Tajima 1989). We used mismatch distributions to evaluate the hypothesis of recent population growth with 99,999 permutations as implemented in ARLEQUIN 3.0.1 (Rogers and Harpending 1992). This distribution is commonly unimodal in populations that have passed through a recent demographic expansion, and is multimodal in stable populations.

\section{Results and Discussion}

The taxonomic classification of Mahseer species has often led to ambiguity; sequencing of the COI gene region of Tor torwill provide valuable genetic information that can be used to solve the ambiguity. Mitochondrial DNA (mtDNA), owing to its unique attributes such as uniparental inheritance, exclusive presence of displacement loop, lack of recombination, high mutation rate, small size, and constant gene content, has recently come to the foreground in the filed of evolutionary genomics. Furthermore, Kraytsberg et al. (2004) stated that mtDNA recombinants fall into two structural classes: class 1, with a short paternal sequence inserted into a mostly maternal molecule, and class 2 , with a maternal sequence flanked by paternal sequences. Therefore, mitochondrial DNA can play a pivotal role in exploring population structure and phylogenetic relationship within Tor tor, which could be of great assistance in species identification and conservation.

It was observed that of the 32 specimens collected, nine specimens from two sites exhibited close association and the other 23 specimens collected from the other six sites exhibited similar mitochondrial DNA sequences, based on the $C O I$ gene sequence analysis for species identification and phylogenetic relationship. Analysis of the aligned COI gene sequences identified 3 haplotypes (Hap_1 to Hap_3); the 3 haplotypes were shown in all of the populations collected from eight sites. Number of specimens belonging to each haplotype is shown in table 1. Specimens from five sites, Chambal river (CR), Rana Pratap Sagar (RPS), Bassi dam (BD), Kali Sindh river (KSR), and Parwan river (PR), exhibited Hap_1; Hap_2 was observed only in specimens obtained from Badi lake; Hap_3 was observed in specimens obtained from Daya dam and Madar tank. Haplotype network analysis (Fig. 2) shows three distinct clades originating from a common ancestral haplotype. They were named as group I, group II and group III, which correlate to Hap_1, Hap_2, and hap_3, respectively. Group I and group II sequences were found to be closely associated. Group III sequences showed divergence from group I and group II sequences. This is also depicted graphically in figure 3 , which shows evolutionary divergence over sequence pairs within groups. Haplotype/nucleotide diversity parameters are depicted in Fig.4. Phylogenetic analysis shows that the fish population collected from Madar tank showed distinct genetic diversity compared to the fish populations collected from the other six sites.

Phylogenetic analysis of the mitochondrial sequences using the Neighbour-Joining (NJ) method strongly supported the reciprocally monophyletic status between Tor tor populations. The Neighbour-joining tree constructed using COI gene data showed that the sequences were clustered into two major clades. Different environmental conditions can lead to the emergence of the same species in different geographic locations with different genetic variations. Present-day climate change issues have widespread effects on organisms (Rocha et al., 2017; Wu et al., 2017).

Evolutionary relationship of the sequenced COI genes of the specimens is shown in Fig. 5. The phylogenetic tree was constructed using the UPGMA method and the optimal tree with the sum of branch length (0.02044436). The highest genetic distance was observed between specimens from Bassi Dam and Chambal 
River (0.0202-2.02\%) followed by that between MT and RPS (0.0199-1.99\%). The lowest genetic distance was observed between specimens from Bassi Dam and Badi Lake (0.0026-0.26\%). The Daya Dam has the "closest" genetic distance from Badi Lake as compared to Chambal river, Rana Pratap Sagar Dam, Kali Sindh River, Bassi Dam and Parwan River. This Comparison clearly indicates that fish population of Daya Dam and Madar tank are genetically different from the other six groups. Most of the specimens from different sites were clustered together, which reflects the earlier morphology-based taxonomic assignment.

\section{Conclusion}

The genetic analysis of Tor tor fishes from eight different locations showed the presence of three distinct haplotypes. Of the three haplotypes, haplotype 3 was observed only from two geographical locations, Madar Tank and Daya Dam. Group 3 was the most diverse, and therefore, better equipped to adapt to changing conditions. This indicates that fish from group 3 have contributed to improving the adaptative value of Mahseer in the course of evolution. These adaptations should be exploited while repopulating the fish, which will contribute to better ecosystem management.

\section{Acknowledgements:}

The authors acknowledge that no funding was received for this study.

\section{Declaration of Conflict of Interest:}

The authors declare that they have no conflict of interests.

\section{Authorship contribution statement:}

Sudha Summarwar: Study conceptualization, experimental work, manuscript preparation.

K.P. Yadav: Study supervision, review;

Harendra Kumar: Study supervision, review;

Satya Prakash Tailor: Study supervision, review.

\section{References:}

Anonymous (1976). In: India, national commission on agriculture (1970) issued in 1976. Technical report. Part VIII fisheries.

Crandall, K.A.; Fetzner Jr., J.W.; Lawler, S.H.; Kinnersley, M. and Austin, C.M. (1999). Phylogenetic relationships among the Australian and New Zealand genera of freshwater crayfishes (Decapoda: Parastacidae). Australian Journal of Zoology 47: 199-214.

Crozier, L.G. and Hutchings, J.A. (2014) Plastic and evolutionary responses to climate change in fish. Evol Appl. 7(1): 68-87.

Jayaram, K.C. (1999). The Freshwater Fishes of the Indian Region. Narendra Publishing House, Delhi. $6: 551$.

Khare, P.; Mohindra, V.; Barman, A.S.; Singh, R.K. and Lal, K.K. (2014). Molecular evidence to reconcile taxonomic instability in mahseer species (Pisces: Cyprinidae) of India. Org Divers Evol. 14:307-326.

Levitus, S. (2012). Sea level change results from the IPCC 2007 Report and subsequent results, In Sea level rise and coastal infrastructure: Prediction, risks, and solutions, American Society of Civil Engineers Council on Disaster Risk Management, Monograph No. 6, eds. B. M. Ayyub and M. S. Kearney. 20-27.

Nguyen, TT.; Na-Nakorn, U.; Sukmanomon, S. and ZiMing, C. (2008). A study on phylogeny and biogeography of Mahseer species (Pisces: Cyprinidae) using sequences of three mitochondrial DNA gene regions. Mol PhylogenetEvol. 48: 1223-1231. 
O'Reilly, C.M.; Sharma, S.; Gray, D.K.; Hampton, S.E.; Read, J.S.; Rowley, R.J. and Zhang, G. (2015). Rapid and highly variable warming of lake surface waters around the globe. Geophysical Research Letters. 42: 10773-10781.

Pachauri, R.K.; Allen, M.R.; Barros, V.R.; Broome, J.; Cramer, W.; Christ, R. and van Ypserle, J.P. (2014). Climate Change 2014: Synthesis Report. Contribution of Working Groups I, II and III to the Fifth Assessment Report of the Intergovernmental Panel on Climate Change. Geneva, Switzerland: IPCC.

Pavan-Kumar, A.; Raman, S.; Koringa, P.G.; Patel, N.; Shah, T.; Singh, R.K.; Krishna, G.; Joshi, C.G.; Gireesh-Babu, P. and Chaudhari, A. (2016). Complete mitochondrial genome of threatened Mahseer Tor tor (Hamilton 1822) and its phylogenetic relationship within Cyprinidae family. J Genet. 95(4):853-863.

Sati, J.; Kumar, R.; Kumari, p.; Sahoo.; Rabindar, S.; Patiyal.; Ali, S. and Barat, A.T. (2013). Genetic characterization of Golden mahseer (Tor putitora) populations using mitochondrial DNA markers. 68-74.

Wasko, A.P., C. Martin, C. Oliveira and F. Forresti. (2003). Non-destructive genetic sampling in fish. An improved method for DNA extraction from fish fins and scales. Hereditas 138:161-165.

\section{Hosted file}

table. docx available at https://authorea.com/users/334651/articles/460616-cytochrome-oxidasei-based-haplotype-diversity-of-mahseer-tor-tor-from-eight-sites-in-southern-rajasthan

\section{Hosted file}

figure.docx available at https://authorea.com/users/334651/articles/460616-cytochromeoxidase-i-based-haplotype-diversity-of-mahseer-tor-tor-from-eight-sites-in-southernrajasthan 